\title{
Anwendung von Augentropfen mit Ozon: Eine Fallserie von spontanen Augenerkrankungen bei Tieren und Menschen
}

\author{
Leopoldo Spadea $^{\mathrm{a}}$ Emanuele Tonti $^{\mathrm{a}} \quad$ Andrea Spaterna $^{\mathrm{b}} \quad$ Andrea Marchegiani $^{\mathrm{b}}$ \\ ${ }^{a}$ Department of Medico-Surgical Sciences and Biotechnologies, Sapienza University of Rome, Rom, Italien; beterinary Teaching Hospital, \\ School of Biosciences and Veterinary Medicine, University of Camerino, Camerino, Italien
}

\section{Schlüsselwörter}

Antimikrobielle Wirkstoffe · Hornhautulkus · Keratokonjunktivitis . Ozon

\section{Zusammenfassung}

Konjunktivitis, Keratokonjunktivitis und Hornhautulzera sind häufige Augenerkrankungen, die sowohl bei Menschen als auch bei Tieren oft diagnostiziert werden; die gängige Behandlung besteht in der topischen Anwendung von Augentropfen mit antiinflammatorischen und antibakteriellen Wirkstoffen. Diese Wirkstoffe sind oft von mangelhafter Wirksamkeit, da Infektionen in hypoxischem Gewebe Methicillin-resistente Staphylococcus aureus und Pseudomonas aeru- ginosa enthalten; daher werden neue Mittel für die Behandlung von Augenschmerzen und -entzündungen benötigt. Die Anwendung von Ozon (nach Stabilisierung für die topische Applikation in Form eines Ozonids) könnte bei bestimmten Erkrankungen des vorderen Augensegments sowohl wegen seiner entzündungshemmenden und bakteriziden Wirkung als auch durch Förderung der Gewebereparatur von Nutzen sein. Ozonisierte Öle haben die gleichen Eigenschaften wie gasförmiges Ozon und sind dabei gut verträglich für Gewebe. In der vorliegenden Arbeit berichten wir über die Reparatur- und Regenerationseffekte von ozonisiertem Öl in Liposomen plus Hypromellose (Ozodrop ${ }^{\circledR}$, FB Vision, Ascoli Piceno, Italien) bei 3- bis 4-mal täglicher Instillation zur Behandlung spontaner externer Augenerkrankungen bei Mensch und Tier.

(c) 2019 S. Karger GmbH, Freiburg

\section{Einleitung}

Ozon $\left(\mathrm{O}_{3}\right)$ ist ein gasförmiges Molekül aus drei Sauerstoffatomen, dessen Struktur infolge mesomerer Zustände dynamisch instabil ist [1]. Dank seiner ausgeprägten oxidativen Wirkung ist Ozon allgemein als einer der stärksten bakteriziden, antiviralen und antifungalen Wirkstoffe bekannt [2].

Doch neben dieser oxidativen Wirkung kann Ozon in der richtigen Dosierung auch verschiedene nützliche biochemische Mechanismen anstoßen und das antioxidative System reaktivieren (mit Katalase, Superoxiddismutase, Glutathionperoxidase etc.) [3]. Zudem wird Ozon heute auch klinisch zur Behandlung von chronischen Wunden wie z. B. trophischen und ischämischen Ulzera und diabetesbedingten Wunden eingesetzt. Die positiven Auswirkungen von Ozon auf die Wundheilung könnten sowohl auf die Hochregulierung der Expression von ThrombozytenWachstumsfaktor, transformierendem Wachstumsfaktor $\beta$ und vaskulärem endothelialem Wachstumsfaktor zurückzuführen sein als auch auf die Reduktion bakterieller Infektion, Besserung der dermalen Wundheilungsstörung oder erhöhte Sauerstoffspannung infolge der Ozonexposition im Wundbereich [2]. Ozon ist im gasförmigen Zustand hochgradig reaktiv und nicht immer als topische Behandlung geeignet. In Kochsalzlösung sinkt seine Konzentration sehr schnell; die Kinetik entspricht hierbei einer

\section{KARGER}

Fax +497614520714

information@karger.com

www.karger.com (c) 2019 S. Karger GmbH, Freiburg

Accessible online at:

www.karger.com/kop
Leopoldo Spadea, MD

Department of Medico-Surgical Sciences and Biotechnologies

Sapienza University of Rome

Via Benozzo Gozzoli 34, 00142 Rom, Italien

leopoldo.spadea@uniroma1.it 
1

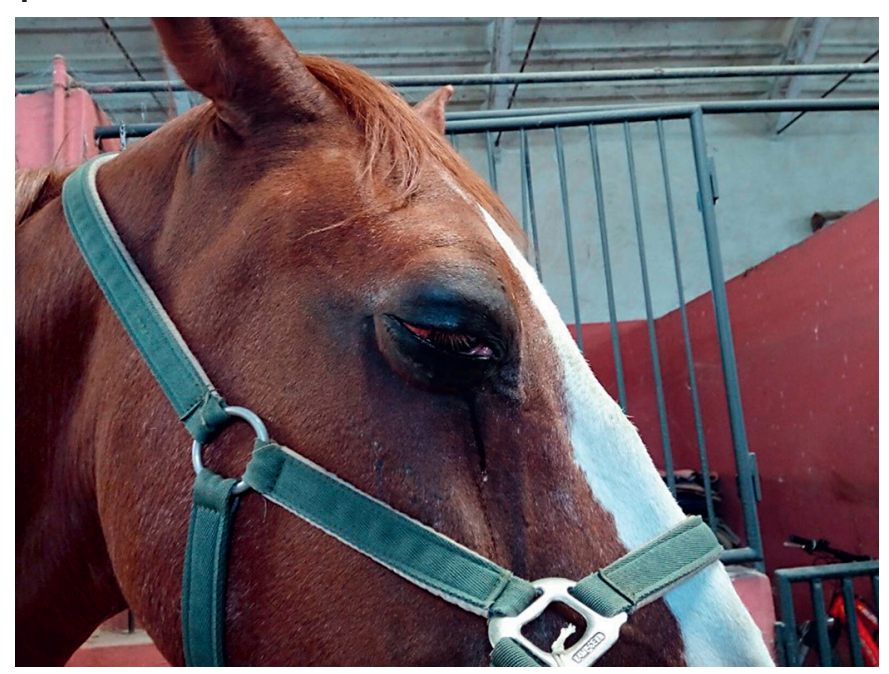

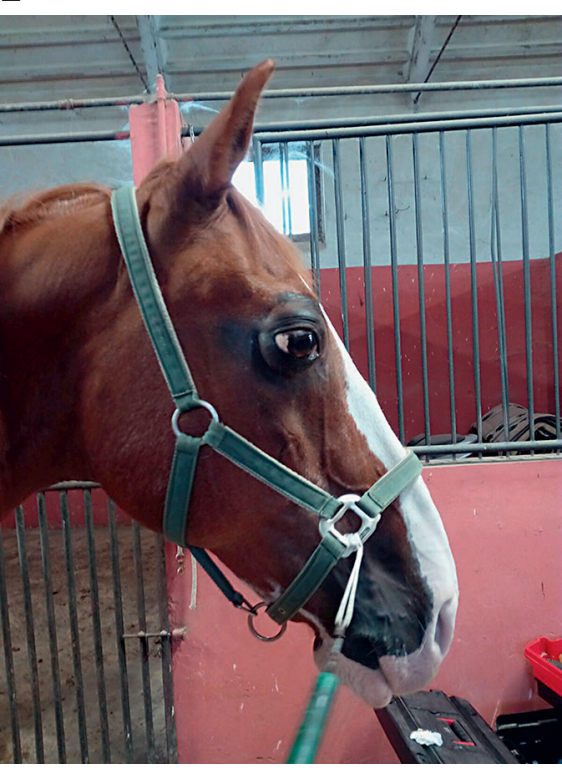

Abb. 1. Rechtes Auge eines 26-jährigen Pferdes vor Behandlungsbeginn. Zu sehen sind Blepharitis und Blepharospasmus, ödematöse und hyperämische Bindehaut und abundanter muköser Augenausfluss.

Abb. 2. Dasselbe Auge wie in Abb. 1 nach 7-tägiger Behandlung. Auge unauffällig.

Reaktion erster Ordnung, und die Halbwertszeit beträgt 2 h. Das bedeutet, dass nach rund $24 \mathrm{~h}$ nur noch sehr wenig Ozon in der Lösung vorliegt. Doch trotz dieser Instabilität lässt sich das Ozonmolekül - zur topischen Anwendung - stabilisieren: als Ozonid zwischen den Doppelbindungen einer einfach ungesättigten Fettsäure wie z. B. Ölsäure $[4,5]$. Aktuelle Anwendungsgebiete von ozonisiertem Öl umfassen die topische Behandlung von Wunden, anaeroben Infektionen, Herpesinfektionen (HSV I und II), trophischen Ulzera und Verbrennungen, Cellulitis, Abszessen, Analfissuren, Dekubitusgeschwüren («Wundliegen»), Fisteln, Pilzerkrankungen, Furunkulose, Gingivitis und Vulvovaginitis [6].

Die Anwendung von Ozon bei bestimmten Erkrankungen des vorderen Augensegments könnte sowohl wegen seiner entzündungshemmenden und bakteriziden Wirkung von Nutzen sein als auch durch Förderung der Gewebereparatur. Ungünstigerweise reizt ozonisiertes Öl in hohem Maße das Hornhautgewebe; daher wurde kürzlich eine Formulierung speziell für die Anwendung am Auge entwickelt, die auf ozonisiertem liposomalem Sonnenblumenöl plus Hypromellose basiert (Ozodrop ${ }^{\circledR}$, FB Vision, Ascoli Piceno, Italien), welches sich mit dem empfindlichen Gewebe der Augenoberfläche sehr gut verträgt.

Unserer Erfahrung nach wird diese speziell für die Anwendung am Auge entwickelte neue ozonisierte liposomale Ölformulierung angewendet, um die Wundheilung zu fördern und verschiedene Infektionsprozesse im Rahmen verbreiteter Augenerkrankungen bei Mensch und Tier zu behandeln. Entzündliche Erkrankungen des vorderen Segments, die eine geeignete antiinflammatorische Therapie erfordern - wie z. B. Konjunktivitis, Keratitis, Sicca-Syndrom oder Hornhautulzera -, machen den Großteil der Augenerkrankungen bei Tieren aus [7] und ha- ben verschiedene Symptome mit solchen Erkrankungen beim Menschen gemeinsam, z. B. Rötung, Chemose und Exsudation. Eine topische Dosierung von einem oder zwei Tropfen Kollyrium alle 4 Stunden über 3-7 Tage wird empfohlen, wobei oft auch mehr Instillationen in kürzeren Abständen erforderlich sind, um klinische Abheilung zu bewirken, und ein solches Therapieschema ist mit der Gefahr verbunden, dass ein Teil der Patienten sich nicht durchgängig daran hält $[7,8]$. Die derzeit verfügbaren Wirkstoffe sind recht teuer und oft von mangelhafter Wirksamkeit, da Infektionen in hypoxischem Gewebe Methicillin-resistente Staphylococcus aureus und Pseudomonas aeruginosa enthalten; in einigen Fällen sind die Wirkstoffe unzureichend und können inakzeptable Nebenwirkungen hervorrufen.

Besonders ausgeprägt ist der Bedarf nach neuen Mitteln zur Behandlung von Augenschmerzen und -inflammation, wie sie bei externen Augeninfektionen und -entzündungen auftreten, da dies mit dem Risiko der Erblindung verbunden ist. Der übermäßige Gebrauch von Antibiotika in der Behandlung von Infektionskrankheiten und die Entwicklung multiresistenter Bakterienstämme haben antimikrobielle Wirkstoffe aus ätherischen Ölen vermehrt in den Mittelpunkt des Forschungsinteresses gerückt [9]. Ozonisierte Öle haben die gleichen Eigenschaften wie gasförmiges Ozon, sind für biologische Gewebe gut verträglich (die Verträglichkeit für die Hornhaut wird durch eine liposomale Formulierung erhöht), und ihre biologischen Wirkungen stehen im Zusammenhang mit Sauerstoffverbindungen [9]. Sie erreichen die Elimination von Pathogenen durch direkte Oxidation unter Vermittlung von Wasserstoffperoxid und Lipoperoxyd sowie selektive Zytotoxizität gegenüber Zellen mit hoher Teilungsrate. Dies geschieht durch Lyse und Zelltod der Bakterien, Herunterregulieren der mitochondrialen Aktivität der Bakterien und Stö- 
rung viraler lytischer Enzyme, mit Überlagerungen ähnlich wie bei den phagozytären Komponenten des Immunsystems [10]. Zugleich fördert Ozon eine "physiologische» Wundheilung und minimiert so das Risiko der Keloidbildung und auch die Gefahr einer Hornhauttrübung.

Darüber hinaus fördert Ozon die Adaptation der Zellen an oxidativen Stress und hemmt pathophysiologische Ereignisse, die durch reaktive Sauerstoffspezies verursacht werden. Eine oxidative Vorbehandlung mit Ozon senkt die Malondialdehydkonzentration signifikant und steigert die Aktivität von Superoxiddismutase, Glutathionperoxidase und Katalase. Die Interleukin-1 $\beta$ Konzentration im Serum sinkt tendenziell unter einer oxidativen Vorbehandlung mit Ozon [10]. Vor dem Hintergrund dieser zahlreichen Eigenschaften und Anwendungsmöglichkeiten von Ozon war das Ziel der vorliegenden Arbeit eine erste Evaluierung der Reparatur- und Regenerationseffekte von ozonisiertem Öl in Liposomen plus Hypromellose (Ozodrop ${ }^{\circledR}$ ) bei 3- bis 4-mal täglicher Instillation zur Behandlung spontaner externer Augenerkrankungen bei Mensch und Tier.

\section{Veterinärmedizinische Fallberichte}

\section{Fall 1}

Ein 26-jähriges, kastriertes, männliches Pferd der Rasse Sella Italiano wurde an die veterinärmedizinische Lehrklinik der Universität Camerino überwiesen; Anlass war ein Exophthalmus des rechten Auges infolge einer retrobulbären Raumforderung (vermutlich Neoplasma/Osteosarkom) in Verbindung mit rezidivierender Konjunktivitis, die mit topischem Antibiotikum (Tobramycin) und sowohl topischen als auch systemischen NSAR (Piroxicam plus Flunixin-Meglumin) behandelt worden war, ohne dass signifikante Besserung eintrat. Am Tag der Vorstellung war das linke Auge des Tiers unauffällig; die Befunde des rechten Auges umfassten Blepharitis und Blepharospasmus, ödematöse und hyperämische Bindehaut und abundanter muköser Augenausfluss (Abb. 1). Am ersten Tag nach Behandlungsbeginn klang der Blepharospasmus vollständig ab, und bei Blepharitis und Bindehautödem war eine Besserung zu erkennen. Auch der Augenausfluss wurde etwas weniger. Nach 3 Behandlungstagen waren Blepharitis und Konjunktivitis vollständig abgeklungen, und nach einer Woche Therapiedauer war das Auge vollständig unauffällig (Abb. 2)

\section{Fall 2}

Bei einer 6 Monate alten männlichen Katze der Rasse Europäisch Kurzhaar (Abb. 3) lag von Geburt an eine chronische Konjunktivitis vor, die auf die gängigen antimikrobiellen und antiinflammatorischen Therapien nicht ansprach. Am Tag der Vorstellung zeigte die Katze ein ausgeprägtes Bindehautödem insbesondere im rechten Auge sowie Rötung der Bindehaut und mukösen Augenausfluss beidseits. Bakteriologische Abstriche der Konjunktiva ergaben im rechten Auge eine normale Bakterienzahl (20 KBE) und das Vorliegen von Staphylococcus spp. und Enterococcus spp. sowie im linken Auge eine erhöhte Keimzahl für Staphylococcus

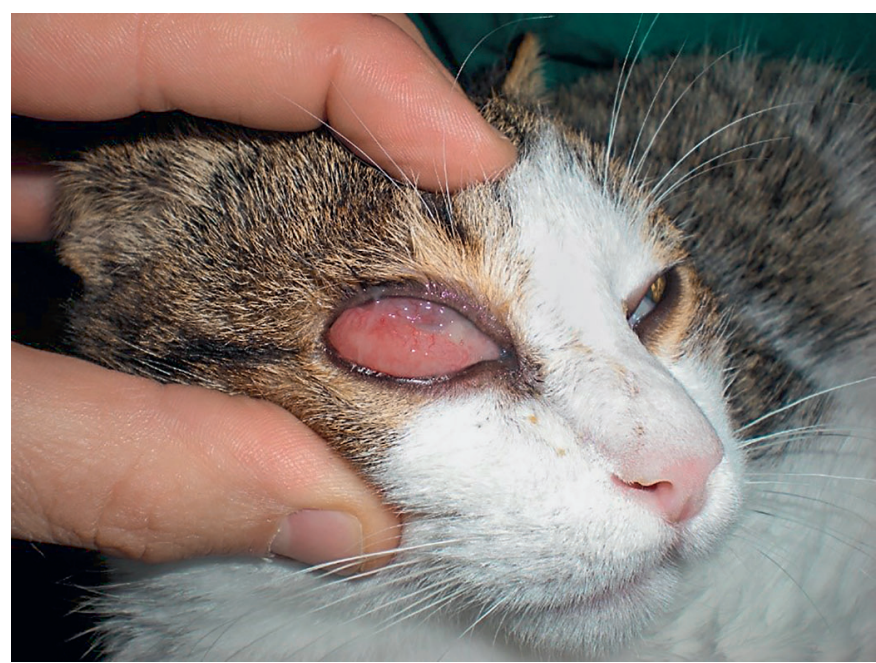

Abb. 3. Rechtes Auge einer 6 Monate alten Katze vor Behandlungsbeginn. Zu sehen sind ein ausgeprägtes Bindehautödem sowie Rötung der Bindehaut und muköser Augenausfluss.

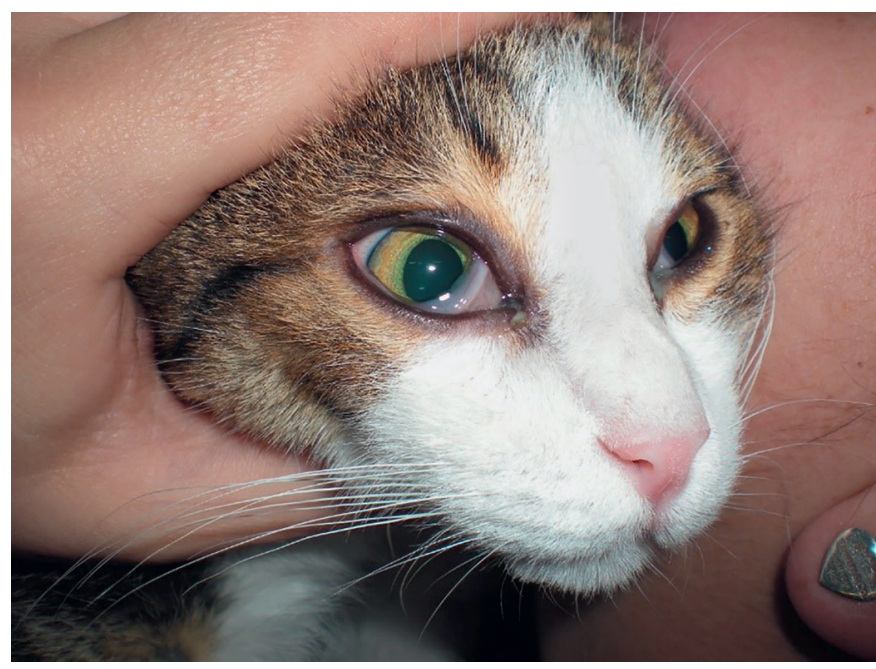

Abb. 4. Dasselbe Auge wie in Abb. 3 nach 10-tägiger Behandlung. Auge unauffällig, bei anhaltendem mukösem Ausfluss.

spp. (140 KBE). Die Therapie war konsistent mit der Instillation von einem Tropfen Kollyrium pro Auge 2-mal täglich bis zur klinischen und bakteriologischen Abheilung. Nach 3 Behandlungstagen war die konjunktivale Keimzahl in beiden Augen normal (20 KBE), und es lagen keine Enterococcus spp. mehr vor. Nach 10 Behandlungstagen waren keine Bindehautsymptome mehr erkennbar (Abb. 4).

\section{Fall 3}

Eine 3-jährige, männliche Englische Bulldogge (Abb. 5) wurde mit einer chronischen Keratitis im rechten Auge infolge von beidseitigem Entropium der Augenlider an unsere Abteilung überwiesen. Aufgrund anderer Probleme unabhängig von der Pathologie, die Anlass für die Überweisung war, konnte der Hund nicht sofort operiert werden, da das Risiko einer Allgemeinanästhesie 


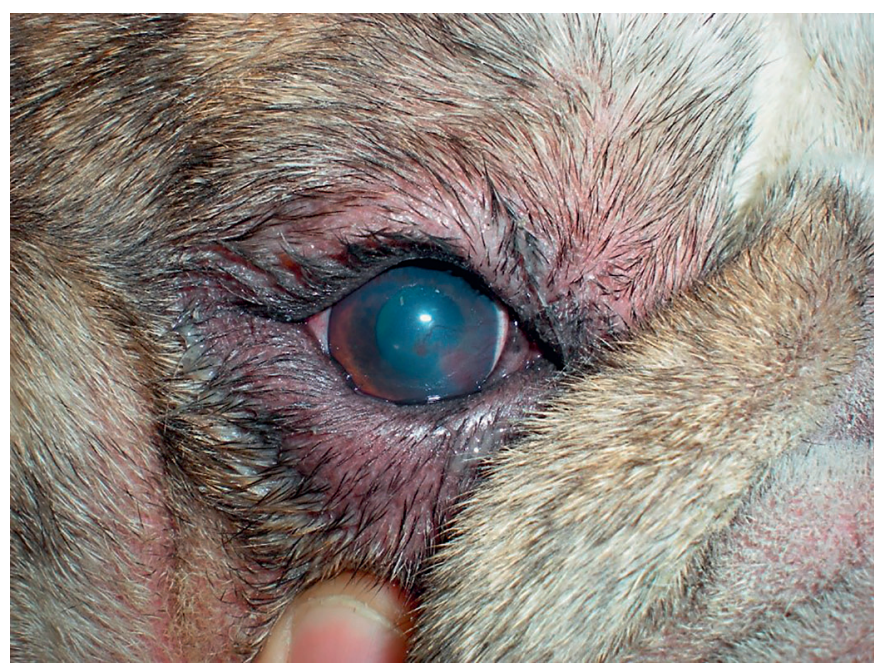

Abb. 5. Rechtes Auge einer 3-jährigen englischen Bulldogge vor Behandlungsbeginn. Zu sehen sind Entropium, muköser Ausfluss, Keratitis, Hornhautödem sowie tiefe und oberflächliche Neovaskularisation.

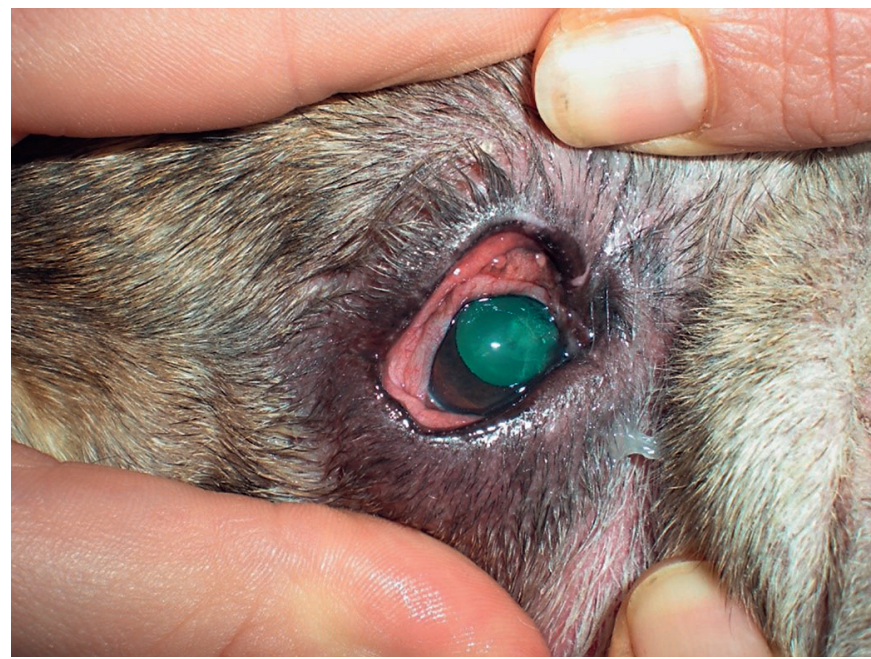

Abb. 6. Dasselbe Auge wie in Abb. 5 nach 10-tägiger Behandlung. Das Hornhautödem ist vollständig abgeklungen, und von der Neovaskularisation verbleibt nur ein einzelnes, kleinkalibriges Gefäßes mit Ursprung am Limbus auf Höhe des Augeninnenwinkels. Entropium und muköser Ausfluss liegen weiterhin vor.

zu hoch war, und in Absprache mit dem Halter wurde beschlossen, die topische Therapie mit der Instillation eines Tropfens Kollyriums zweimal täglich bis zur Operation einzuleiten. Am Tag der Vorstellung zeigte der Hund Keratitis, Hornhautödem sowie tiefe und oberflächliche Neovaskularisation. Befindlichkeitsstörung, Juckreiz und muköser Augenausfluss waren ebenfalls zu erkennen. Nach 10-tägiger Behandlung war die Keratitis fast vollständig zurückgegangen, das Hornhautödem war vollständig abgeklungen, und von der Neovaskularisation verblieb nur noch ein einziges, kleinkalibriges Gefäßes, das auf Höhe des Augeninnenwinkels aus dem Limbus entsprang (Abb. 6). Entropium und muköser Ausfluss lagen weiterhin vor.

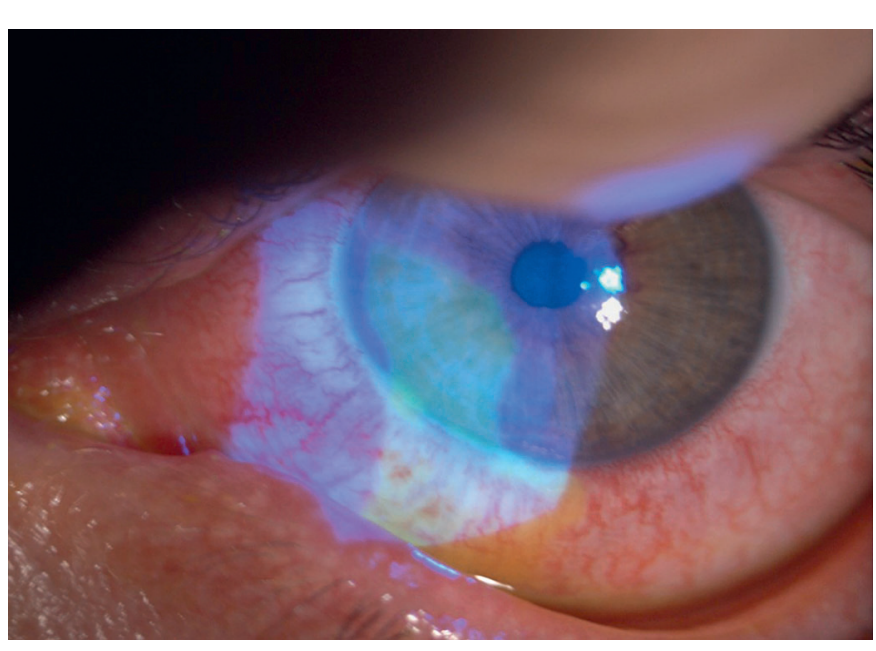

Abb. 7. Linkes Auge eines 33-jährigen Mannes vor Behandlungsbeginn. Zu sehen sind ziliärer Flush und ein umfangreicher Epitheldefekt.

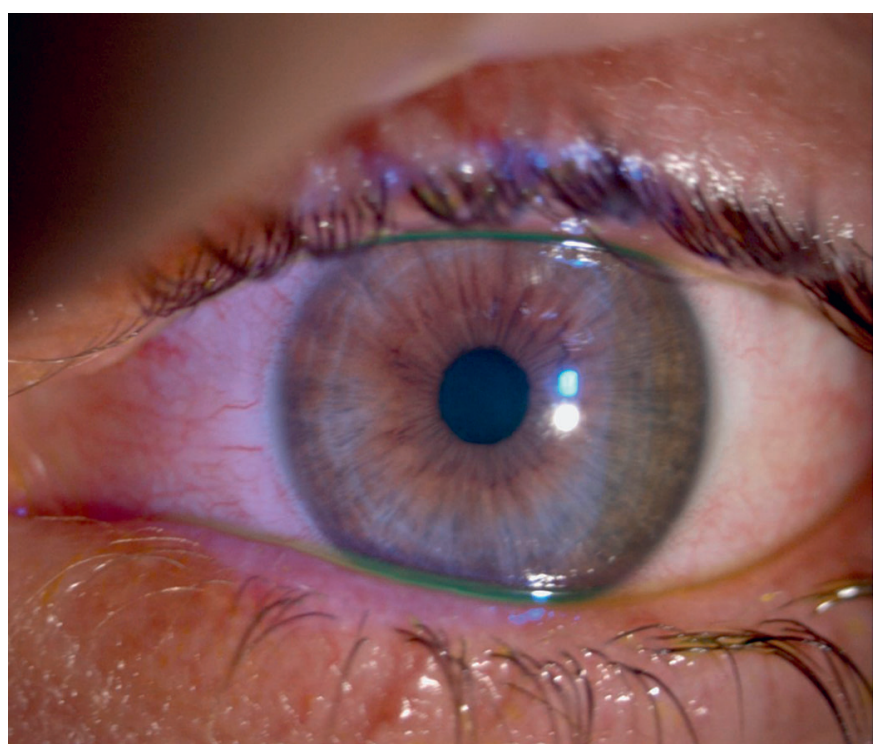

Abb. 8. Dasselbe Auge wie in Abb. 7 nach 3-tägiger Behandlung; das Erscheinungsbild ist das eines gesunden Epithels ohne Anzeichen einer Entzündung.

\section{Humanmedizinische Fallberichte}

\section{Fall 1}

Ein 33-jähriger Mann mit unsachgemäßer Anwendung von Kontaktlinsen einschließlich kontinuierlichen Tragens in der Vorgeschichte stellte sich wegen seit 2 Tagen bestehender Schmerzen, Rötung und Photophobie des linken Auges in unserer Abteilung vor. Der korrigierte Fernvisus betrug 20/25. Die Spaltlampenuntersuchung ergab eine ausgeprägte konjunktivale Gefäßinjektion mit ziliärem Flush sowie eine umfangreiche inferior-nasale Hornhautabrasion mit einem positiven Fluoreszein-Färbungstest (Abb. 7). Der Augeninnendruck lag im normalen Bereich. Die 


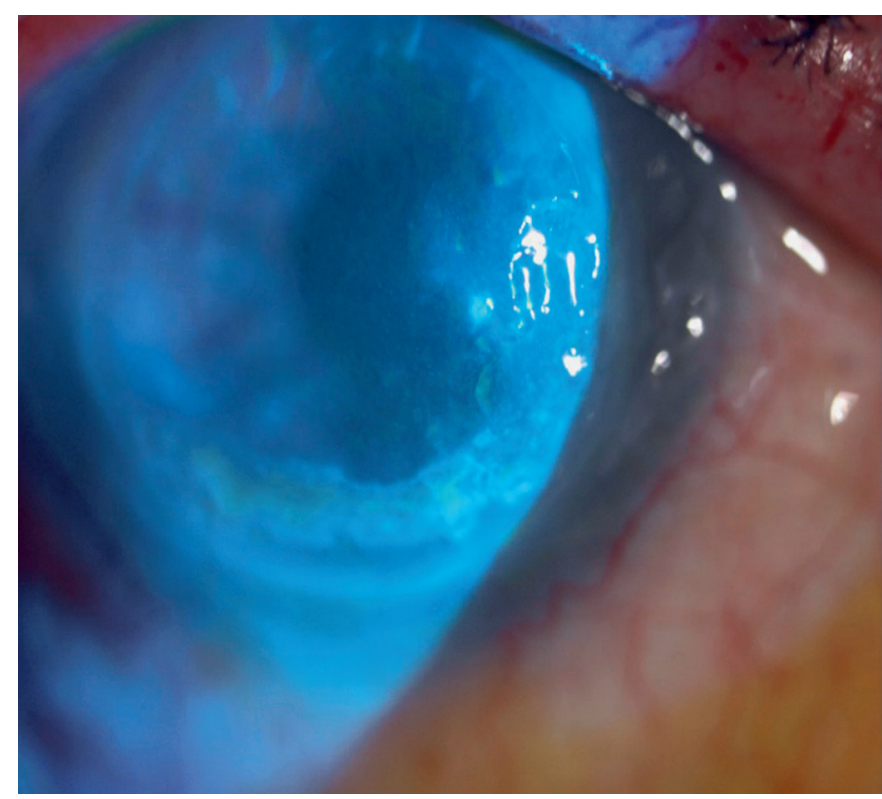

Abb. 9. Rechtes Auge einer 79-jährigen Frau vor Behandlungsbeginn. Zu sehen ist ein Hornhautulkus inferior von der 5-Uhr- bis zur 8-Uhr-Position.

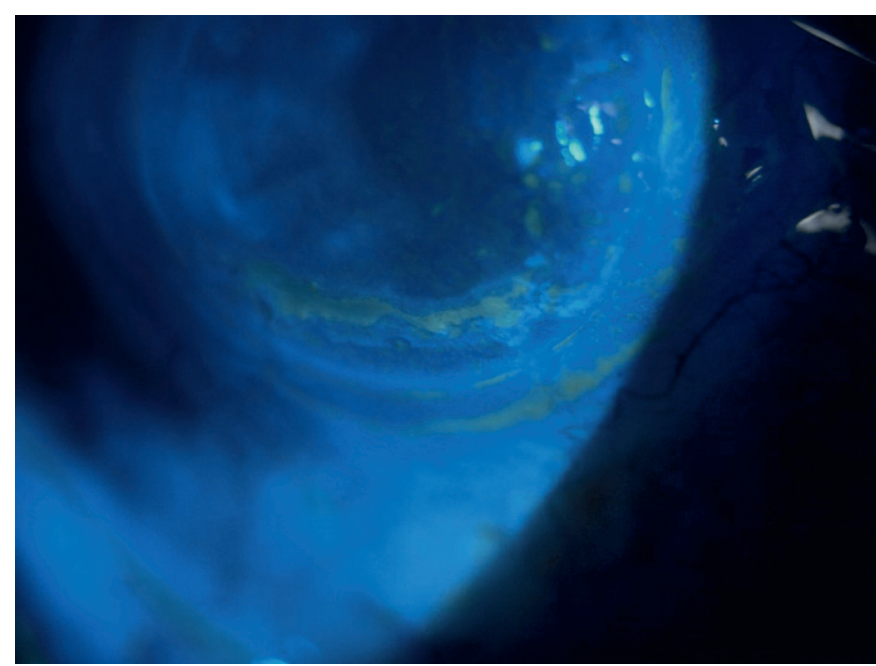

Abb. 10. Dasselbe Auge wie in Abb. 9 nach 7-tägiger Behandlung. Partielle Abheilung des Ulkus mit residuellem cornealem Pooling.

Vorderkammer war reizfrei, und die Pupille war rund und reaktiv. Eine Behandlung mit topischem Moxifloxacinhydrochlorid 0,5\% 3-mal täglich und Ozodrop ${ }^{\circledR}$ 4-mal täglich wurde verordnet. Drei Tage später war der Patient frei von jeglichen Augenbeschwerden, und die Untersuchung ergab eine klare Hornhaut, gute Heilung des Defekts und keine Anzeichen einer Entzündung (Abb. 8).

\section{Fall 2}

Eine 79-jährige Frau stellte sich in unserer Abteilung vor und klagte über Augenschmerzen und -reizung, Kopfschmerzen, Photophobie und Ausfluss im rechten Auge. Die Patientin hatte

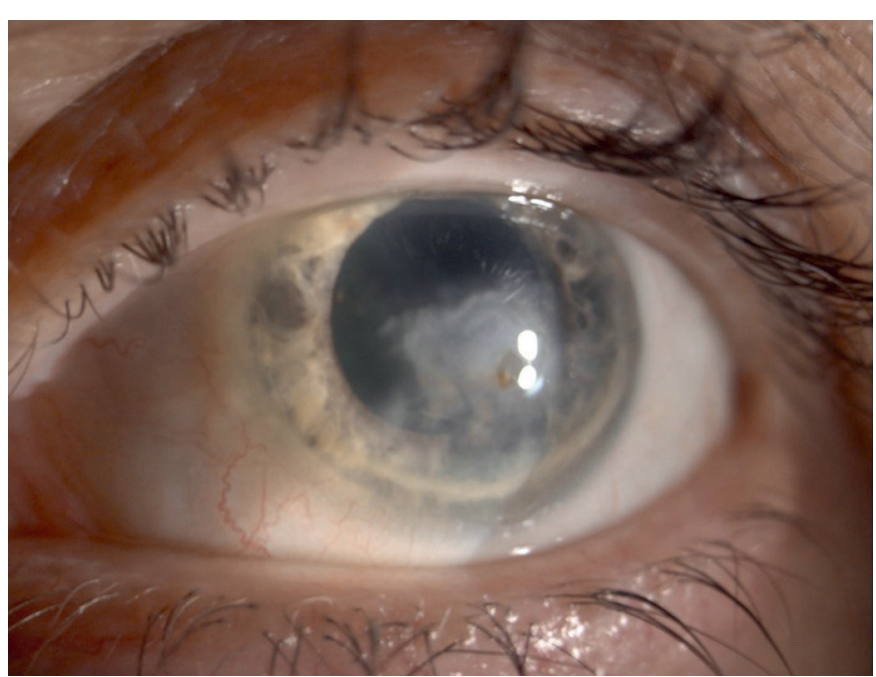

Abb. 11. Linkes Auge eines 71-jährigen Mannes vor Behandlungsbeginn. Zu sehen sind stromale Keratitis und ein Hornhautulkus.

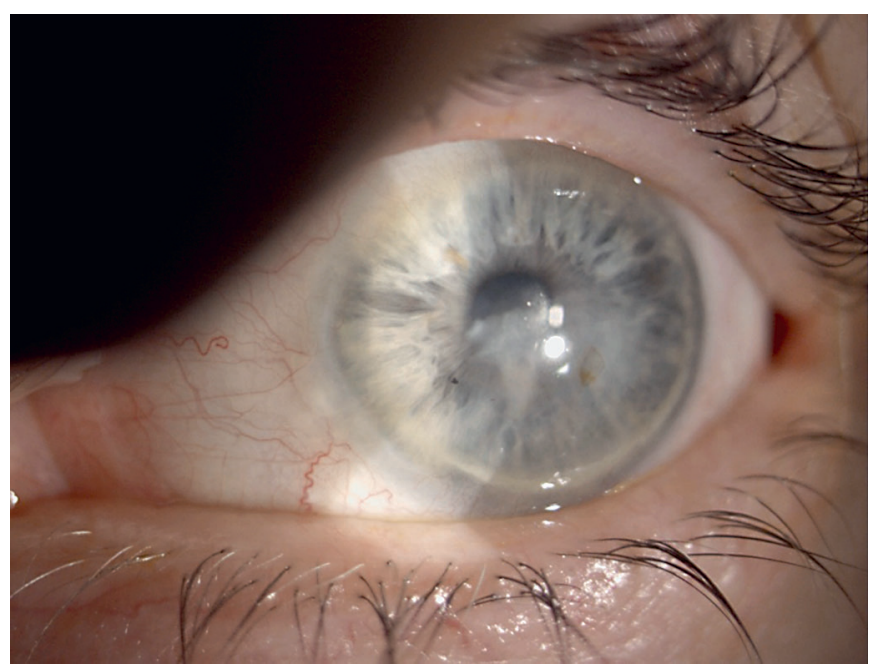

Abb. 12. Dasselbe Auge wie in Abb. 11 nach 10-tägiger Behandlung. Hornhautulkus abgeklungen, Färbung negativ.

sich 10 Jahre zuvor einer perforierenden Keratoplastik unterzogen und war seit 3 Monaten unter Beobachtung wegen einer Band-Keratopathie, die durch ein Hornhautulkus inferior im selben Auge von der 5-Uhr- bis zur 8-Uhr-Position kompliziert wurde. Die Untersuchung ergab periläsionale Infiltrate und Ödembildung mit Färbung unter kobaltblau gefiltertem Licht (Abb. 9). Der Augeninnendruck lag im normalen Bereich. Die Vorderkammer war reizfrei, und die Pupille war rund und reaktiv. Der Patientin wurde eine 7-tägige Kur mit topischer Anwendung von Ozodrop ${ }^{\circledR}$ 4-mal täglich verordnet. Die Untersuchung nach dieser Behandlung ergab eine Besserung der klinischen Symptome und Reduktion des Infiltrats und des Ödems. Darü- 
ber hinaus war eine partielle Abheilung des Ulkus mit residuellem cornealem Pooling unter dem Kobaltblaufilter zu erkennen (Abb. 10). Von den assoziierten Symptomen verblieb nur noch eine leichte Photophobie.

\section{Fall 3}

Ein 71-jähriger Mann wurde wegen Schmerzen und verschwommener Sicht im linken Auge, die auf konventionelle Augentropfen nicht ansprachen, an unsere Abteilung überwiesen. In der Vorgeschichte des Patienten lag ein 2 Monate zuvor aufgetretenes HSVasooziiertes Hornhautulkus vor. Der korrigierte Fernvisus betrug $1 / 20$, bei starker Photophobie und Blendung. Die Untersuchung ergab einen zentralen, prominenten, scheibenförmigen Bereich von Stromakeratitis (Abb. 11) mit einem runden stromalen Infiltrat und Verdünnung der Hornhaut. Darüber hinaus war ein zentrales Hornhautulkus mit positiver Färbung unter kobaltblau gefiltertem Licht zu erkennen. Der Augeninnendruck lag im normalen Bereich. Die Vorderkammer war reizfrei, und die Pupille war rund und reaktiv. Nach 10-tägiger Behandlung mit Ozodrop $^{\circledR}$ 4-mal täglich war der betroffene Hornhautbereich begrenzter (Abb. 12), die Färbungszeichen waren zufriedenstellend abgeklungen, und die Symptome der Photophobie und Blendung waren signifikant verringert.

\section{Diskussion}

Konjunktivitis stellt bei Pferden eine therapeutische Herausforderung dar, da mehrere verschiedene Wirkstoffe angewandt werden müssen, die praktisch nie in einem einzigen Augentropfenpräparat vereint sind, die Pferde jedoch schnell unkooperativ werden, sodass der Tierarzt oft auf andere Behandlungsoptionen ausweichen muss (z. B. subpalpebrale Spülsysteme). Im hier vorgestellten Fall rezidivierender Konjunktivitis infolge einer retrobulbären Läsion war die Behandlung mit den ozonhaltigen Augentropfen erfolgreich. Die Anwendungshäufigkeit von 3-mal täglich wurde vom Tier gut toleriert; es zeigte gute Compliance, und das Behandlungsergebnis hatte auch langfristig Bestand. Bei Katzen ist Konjunktivitis ein häufiges Problem; die gängige Therapie besteht in der topischen und in einigen Fällen sogar systemischen Anwendung von Antibiotika und NSAR. Im hier vorgestellten Fall konnte mit den ozonhaltigen Augentropfen die konjunktivale Kongestion in 10 Tagen reduziert werden. Zudem waren bereits nach nur 3-tägiger Behandlung die zuvor vorhandenen Enterococcus spp. nicht mehr nachweisbar, und die Gesamtkeimzahl war auf das für Katzen als normal geltende Niveau gesunken. Bei brachyzephalischen Hunden stellt Entropium ein verbreitetes Problem dar, und die Hornhaut leidet oft unter daraus resultierenden Veränderungen wie Erosion/Ulzeration, Inflammation, Neovaskularisation und Pigmentierung. Die Behandlung des Entropiums erfolgt in der Regel operativ und erfordert eine Allgemeinanästhesie. In Fällen wie dem hier vorgestellten, in dem nicht sofort operiert werden konnte, ließen sich mit den ozonhaltigen Augentropfen die Beschwerden des Hundes verringern und die Hornhautgesundheit verbessern, ob- wohl die zugrunde liegende Ursache (das mechanische Schleifen der Wimpern auf der Hornhaut) weiterhin vorlag.

Die mikrobielle Keratitis beim Menschen ist ein ophthalmologisches Problem der öffentlichen Gesundheit, da sie weltweit eine der Hauptursachen für Erblindung und Sehbehinderungen darstellt und mit erheblichen wirtschaftlichen Auswirkungen einhergeht. Risikofaktoren wie das Tragen von Kontaktlinsen, Traumata, intraokulare Chirurgie, Erkrankungen der Augenoberfläche und systemische Erkrankungen können die mikrobielle Adhäsion und Infektionen begünstigen. Seit einigen Jahren wird angesichts der Zunahme antibiotikaresistenter Bakterien die prospektive Forschung zur Entdeckung neuer antimikrobieller Strategien vorangetrieben. Der Literatur zufolge könnte KollagenQuervernetzung (CXL) eine alternative Option für refraktäre Fälle von HSV und bakterieller Keratitis beim Menschen sein. Die durch die CXL erzeugten reaktiven Sauerstoffspezies können die Vermehrung von Pathogenen ganz oder teilweise unterdrücken, indem sie deren Nukleinsäuren zerstören [11]. Aktuelle Ergebnisse sprechen dafür, dass CXL eine akzeptable komplementäre Methode zur Behandlung der infektiösen Keratitis sein könnte [12]. Dank seiner starken oxidativen Wirkung könnte das - zur topischen Anwendung stabilisierte - Ozonmolekül eine sinnvolle und einfachere Alternative darstellen. Unsere Erfahrungen scheinen die Sicherheit, Wirksamkeit und Nebenwirkungsfreiheit dieses therapeutischen Vorgehens zu bestätigen.

Unsere ersten Betrachtungen von in-vivo-Fällen belegen, dass ozonhaltige Augentropfen antiinflammatorische und bakterizide Aktivität besitzen und zusätzlich die Gewebereparatur fördern. Alle diese vorteilhaften therapeutischen Effekte werden mit nur einem Augenpräparat erzielt, wodurch das Risiko der mangelnden Kooperation des Patienten bei der Anwendung verringert wird. Ihre Vielseitigkeit und die vielfältigen Anwendungsmöglichkeiten in der Ophthalmologie machen ozonhaltige Augentropfen zu einer sehr attraktiven Option.

Als Fazit unserer ersten Ergebnisse lässt sich sagen, dass ozonhaltige Augentropfen einen validen und geeigneten alternativen Ansatz für die Behandlung externer Augenkrankheiten bei Mensch und Tier darstellen. Darüber hinaus können ozonhaltige Augentropfen wegen ihrer antimikrobiellen Eigenschaften potenziell auch unterstützend in der Vorbereitung auf intra- und extraokulare chirurgische Eingriffe eingesetzt werden (z. B. vor einer Kataraktoperation oder intravitrealen Injektion). Es sind jedoch weitere Studien erforderlich, um diese Ergebnisse zu bestätigen.

\section{Erklärung zu ethischen Konflikten}

Die Studie wurde von der Ethikkommission der Universität genehmigt.

\section{Disclosure Statement}

Die Autoren haben keine finanziellen oder proprietären Interessen an den in dieser Studie beschriebenen Materialien. 


\section{Literatur}

1 Elvis AM, Ekta JS. Ozone therapy: A clinical review. J Nat Sci Biol Med. 2011 Jan;2(1):66-70.

$\checkmark 2$ Kim HS, Noh SU, Han YW, Kim KM, Kang H, Kim HO et al. Therapeutic effects of topical application of ozone on acute cutaneous wound healing. J Korean Med Sci. 2009 Jun;24(3):368-74.

-3 Bocci VA, Zanardi I, Travagli V. Ozone acting on human blood yields a hormetic dose-response relationship. J Transl Med. 2011 May;9(1):66.
4 Valacchi G, Fortino V, Bocci V. The dual action of ozone on the skin. Br J Dermatol. 2005 Dec;153(6):1096-100.

5 Bocci V. Oxygen-ozone therapy: a critical evaluation. Dordrecht: Kluwer Academic Publisher; 2002.

6 Bocci V. Ozone: A new medical drug. Dordrecht: Springer; 2005.

7 Hendrix DV. Diseases and surgery of the canine conjunctiva and nictitating membrane. Hoboken: Blackwell Publishing; 2007.

8 Baeyens V, Felt-Baeyens O, Rougier S, Pheulpin S, Boisramé B, Gurny R. Clinical evaluation of bioadhesive ophthalmic drug inserts (BODI) for the treatment of external ocular infections in dogs. J Control Release. 2002 Dec;85(1-3):163-8.
9 Sechi LA, Lezcano I, Nunez N, Espim M, Duprè I, Pinna A et al. Antibacterial activity of ozonized sunflower oil (Oleozon). J Appl Microbiol. 2001 Feb;90(2):279-84.

10 Bardi JS. News and views. Scripps Research Institute; 2002.

11 Tabibian D, Mazzotta C, Hafezi F. PACK-CXL: corneal cross-linking in infectious keratitis. Eye Vis (Lond). 2016 Apr;3(1):11.

12 Khalili MR, Jahadi HR, Karimi M, Yasemi M. Corneal collagen cross-linking for treatment of bacterial and herpetic keratitis. J Clin Diagn Res. 2017 Jul;11(7):NC12-6. 

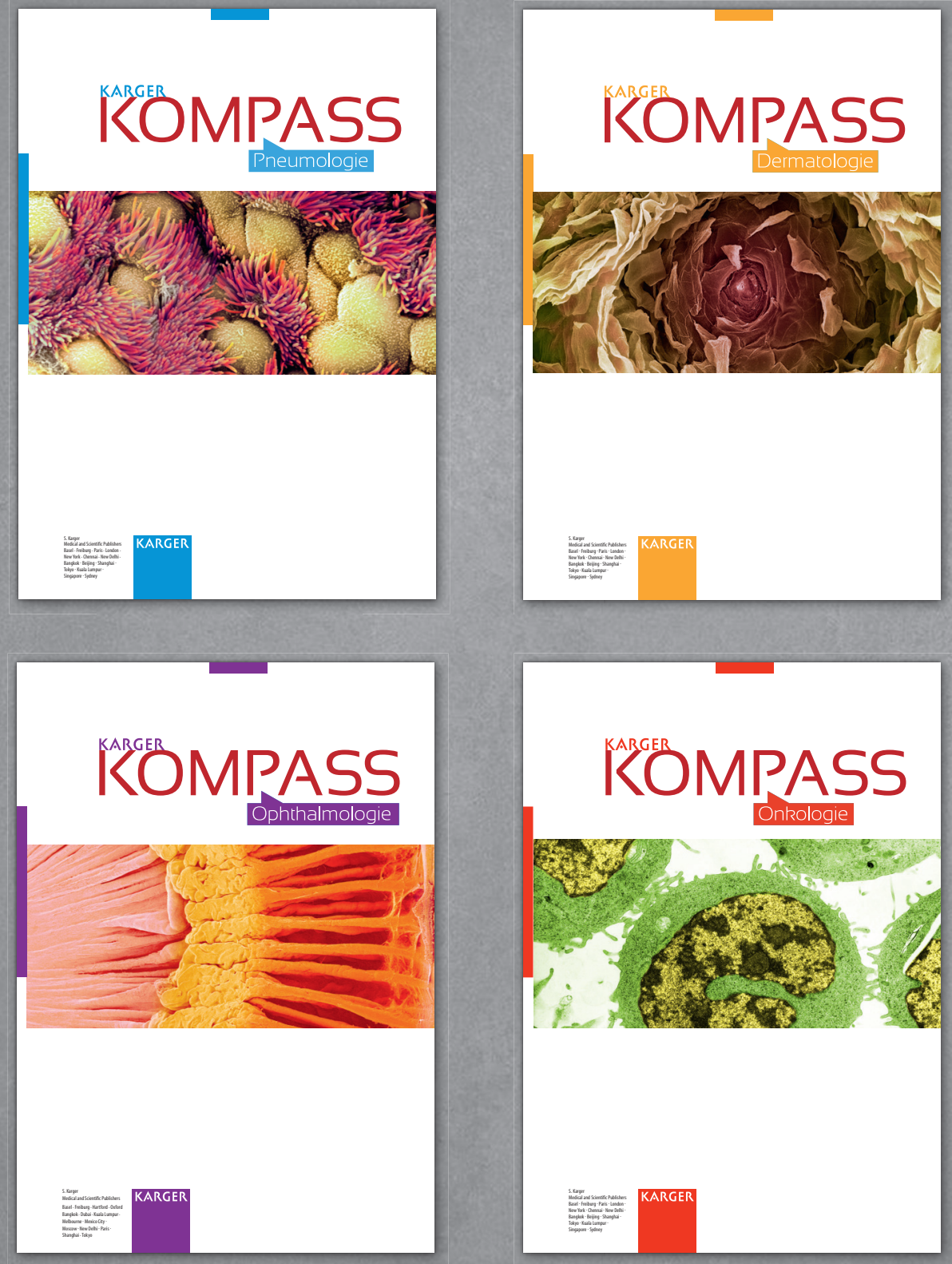

KaRGER KompasS - für Kliniker und niedergelassene Fachärzte. Evidenzbasierte Medizin ist die Basis für informierte Entscheidungen in der Gesundheitsversorgung. Im KaRGER Kompass erhalten Sie Überblick und Orientierung zugleich. Ausgesuchte Übersichtsarbeiten und Fallberichte aus internationalen Fachzeitschriften führen in die Schwerpunkte der Themenhefte ein. In den einzelnen Wissenstransfer-Artikeln lesen Sie über Forschungsergebnisse - kritisch betrachtet und immer mit Blick auf den Patienten und den Nutzen für die Praxis. Unser Service für Sie: Alle Übersichten und Fallberichte sind ins Deutsche übersetzt. Im «Campus» finden Sie ein Forum für fachliche und berufspolitische Themen. Im «Steckbrief Forschung» erläutern junge Wissenschaftler ihre prämierten Forschungsarbeiten. Sorgfältig recherchierte Kurzmeldungen zu gesundheitspolitischen und fachlichen Entwicklungen runden den KARGER Kompass ab.

Bestellen Sie unverbindlich ein kostenloses Probeheft

S. Karger Verlag für Medizin und Naturwissenschaften GmbH · Wilhelmstraße 20A · 79098 Freiburg (Deutschland) Telefon: +49 761 45207-0 ·Fax:+49 761 45207-14 · E-Mail: information@karger.com 